\title{
Robot-assisted stapedotomy: Micropick fenestration of the stapes footplate
}

\author{
DANIEL L. ROTHBAUM, MD, JAYDEEP ROY, PhD, DAN STOIANOVICI, PhD, PETER BERKELMAN, PhD, GREGORY D. HAGER, PhD, \\ RUSSELL H. TAYLOR, PhD, LOUIS L. WHITCOMB, PhD, HOWARD W. FRANCIS, MD, and JOHN K. NIPARKO, MD, \\ Baltimore, Maryland
}

OBJECTIVE: Micropick fenestration of the stapes footplate, a difficult step in stapedotomy, was selected for trials evaluating the potential for robotic assistance (RA) to improve clinical measures of surgical performance.

STUDY DESIGN: In a surgical model of stapedotomy, we measured accuracy of fenestration to a desired point location and force applied to the stapes footplate. Performance variables were measured for 3 experienced and 3 less-experienced surgeons.

RESULTS: RA significantly reduced the maximum force applied to the stapes footplate. For fenestration targeting, RA significantly improved accuracy for less-experienced surgeons and significantly worsened targeting for more-experienced surgeons.

CONCLUSIONS: RA significantly improves performance for micropick fenestration in a surgical

From the Departments of Otolaryngology-Head and Neck Surgery (Drs Rothbaum, Francis, and Niparko), Mechanical Engineering (Drs Roy, Stoianovici, and Whitcomb), Computer Science (Drs. Berkelman, Hager, and Taylor), and Urology (Dr Stoianovici).

Presented at the Annual Meeting of the American Academy of Otolaryngology-Head and Neck Surgery, San Diego, CA, September 22-25, 2002

We gratefully acknowledge the following organizations for their support of this research: American Academy of Otolaryngology-Head and Neck Surgery Foundation Resident Research Grant, NIH Resident Training Grant, National Science Foundation grants IIS9801684 and EEC9731478, The Dexter F. and Dorothy H. Baker Foundation, and The Sidgmore Family Foundation. We also thank the following companies for their support: Medtronic-Xomed, Storz Instruments, Smith and Nephew Instruments, Sawbones Corporation, Dentsply Caulk Corporation, Harwick Chemical, Jeneric/Pentron Corporation, Kerr Corporation, Stryker Leibinger, Dow Corning, and Ferro Corporation.

Reprint requests: Daniel L. Rothbaum, MD, Department of Otolaryngology-Head and Neck Surgery, JHOC 6252, Johns Hopkins Hospital, 601 North Caroline St, Baltimore, MD 21287; e-mail, rothbaum@ robotics.me.jhu.edu.

Copyright (C) 2002 by the American Academy of Otolaryngology-Head and Neck Surgery Foundation, Inc.

0194-5998/2002/\$35.00 + 0 23/1/129729

doi:10.1067/mhn.2002.129729 model of stapedotomy. For certain tasks, RA differentially affects performance for users of different experience levels.

CLINICAL SIGNIFICANCE: These are the first results showing quantitative improvements in performance during simulated ear surgery using RA and differential effects of RA on performance for users of different experience levels. (Otolaryngol Head Neck Surg 2002; 127:417-26.)

$\mathbf{T}$ hroughout the twentieth century, otologic surgery has been transformed by a number of technologic advances in visualization, instrumentation, and intraoperative and post-operative monitoring. Developments such as the stereomicroscope and surgical drill have addressed challenges inherent in a constrained operative field with limited viewing angles. Schuknecht ${ }^{1}$ noted that otologic surgery poses particular challenges in requiring viewing of an instrument's tip along its axis. Although surgeons possess greater manual dexterity than nonsurgeons, human limitations in dexterity $^{2}$ and tactile sensitivity ${ }^{3}$ continue to complicate and constrain microsurgical procedures.

Recent developments in computers and robotics promise to overcome natural human limitations in both dexterity (tremor, jerk, drift, and overshoot) ${ }^{4}$ and tactile sensitivity. Thus, robotic devices offer the possibility of extending human performance to permit fine manipulation tasks that are normally considered difficult or impossible. By enhancing surgical dexterity, robotic assist devices might improve surgical outcomes and enable new procedures currently considered unfeasible. In otologic surgery, robotic devices have already been developed for estimation of stapes footplate thickness 5 and autonomous fenestration of the stapes footplate with a microdrill. ${ }^{6}$

Various designs of robotic assist devices exist for augmenting human task performance. "Extenders" are robotic extensions of the operator's body and are used for tasks beyond normal human strength, such as handling heavy cargo and ma- 
Tactile Feedback Modes: Force-feedback (1:1) vs. Force-scaling (2:1)

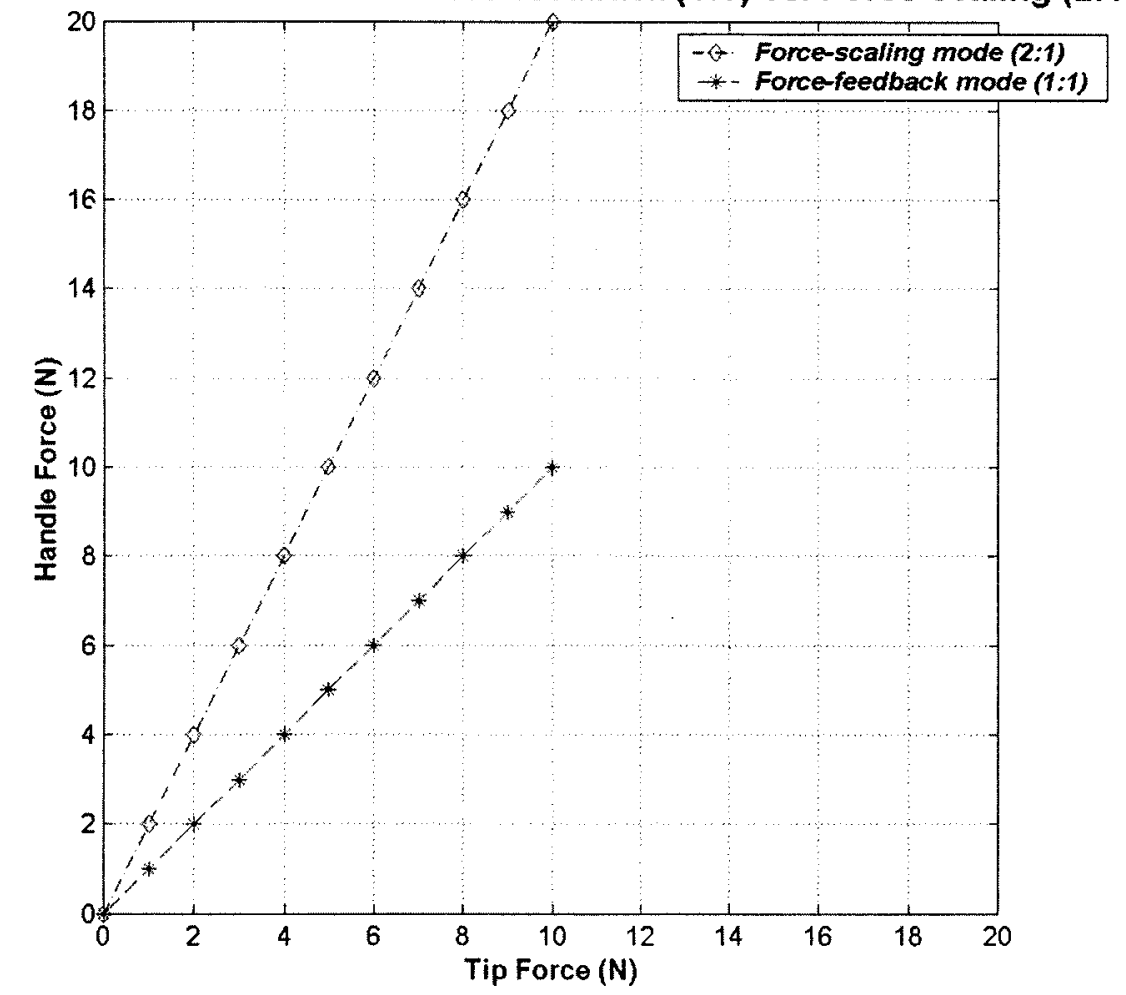

Fig 1. Tactile feedback modes. In force-feedback mode, the force sensed by the surgeon at the instrument handle equals the tip force. There is a 1:1 ratio between handle and tip forces. In force-scaling mode, the SH robot provides amplified haptic feedback. At a force-scaling factor of $n: 1$, the force felt by the surgeon is $n$ times the tip contact force. For these trials, a force-scaling ratio of 2:1 was used, meaning $1 \mathrm{~N}$ at the tool tip feels like $2 \mathrm{~N}$ at the handle.

neuvering heavy loads. ${ }^{7}$ Passive devices "direct" human task execution by enforcing boundaries that limit operator-controlled robot movements. Passive devices are useful in setting limits for safety and precision. ${ }^{8}$ Active devices, such as the Steady Hand ( $\mathrm{SH}$ ) robot, described herein, function as an integrated component of the surgical procedure and thus directly affect fine manipulation tasks. They are useful for selectively amplifying (or reducing) elements of human motor and sensory performance. ${ }^{9}$ For instance, the SH robot can both reduce tremor and amplify tactile sensation. ${ }^{9}$

In the SH robotic system, the operator co-manipulates the surgical tool in cooperation with a robot arm. The surgeon and robot "co-hold" the instrument. The surgeon holds and manipulates the instrument at the "instrument handle." The robot senses the forces exerted by the surgeon on the instrument handle and synthesizes this information along with the sensed tool-tip forces to provide tremor-free positional control. ${ }^{9}$

The SH robot dampens high-frequency movement (ie, tremor) by acting as a viscous system. Physiologic tremor typically manifests as $10-\mathrm{Hz}$ sinusoidal movements and along with low-frequency drift has been shown to affect task performance for microsurgeons. ${ }^{2}$ With the SH robot, the surgeon feels like he or she is moving the instrument in a viscous fluid. For certain tasks, the SH robot has been shown to enhance dexterity. ${ }^{10}$

At the present the $\mathrm{SH}$ robot can be operated in 2 different modes (Fig 1).

In the force-feedback mode ( $\mathrm{SH}$ at 1:1 forcefeedback), the robot provides $1: 1$ haptic feedback to the surgeon. With 1:1 haptic feedback, forces felt by the surgeon at the instrument handle correspond exactly to the contact forces between the tool tip and environmental surface. In other words, there is a 1:1 ratio between the handle and tool-tip forces. 
In the force-scaling mode ( $\mathrm{SH}$ at $\mathrm{n}: 1$ forcescaling), the robot provides amplified haptic feedback. At a force-scaling factor of $n: 1$, the force felt by the surgeon is n-times the contact force between the tool-tip and environmental surface. For all trials reported herein, a force-scaling factor of 2:1 was used. At a force-scaling factor of $2: 1,1 \mathrm{~N}$ at the tool-tip feels like $2 \mathrm{~N}$ at the handle. Force scaling increases the user's ability to feel tool-tip contact forces.

As a first step in developing robotic assistance devices for otologic surgery, we have applied the $\mathrm{SH}$ robotic system to a mechanical model of stapedotomy, a technically demanding procedure presenting operative challenges generally encountered in middle ear surgery. Stapedotomy is an attractive model procedure for developing and testing robotic assist technologies that can be used more generally in other middle ear procedures. Our goal is to develop robotic assist devices for middle ear surgery and to assess their use in simulated surgical trials.

Although a well-established procedure, stapedotomy might benefit from the ability of robotic devices to enhance dexterity and tactile sensitivity. According to guidelines proposed by the Committee on Hearing and Equilibrium, primary stapedotomy has a reported success rate of approximately 70\%.11 Complications associated with stapedotomy typically result from either cochlear or labyrinthine trauma. As manifested by decreases in pure tone thresholds and speech discrimination scores, cochlear trauma leads to sensorineural hearing loss in $5 \%$ to $15 \%$ of patients. Vertigo occurs in approximately $2 \%$ of patients. ${ }^{12}$

For stapedotomy, surgical skill is among the most important variables predicting outcome. ${ }^{13} \mathrm{In}$ fact, some authors submit that only surgeons with significant experience and frequent opportunity to perform this procedure should perform the operation. ${ }^{13}$ Two steps in the stapedotomy operation are particularly demanding: 1) fenestration of the stapes footplate and 2) crimping of the stapes prosthesis to the incus. ${ }^{14,15} \mathrm{We}$ apply the $\mathrm{SH}$ robot to one of those steps, fenestration, to measure the impact of robot assistance. Our goal is to improve task performance for micropick fenestration so that we might reduce complications and obtain better success rates for stapedotomy.

\section{METHODS}

The goal of this investigation is to evaluate the efficacy of SH robot assistance by comparing performance measures of stapedotomy performed (1) manually and (2) with robotic assistance and, further, to evaluate subspecialty expert/novice differences in the performance of micropick fenestration of the stapes footplate. Expert surgeons were defined as full-time otologic surgeons ( $\mathrm{Sr}$ ), and novice surgeons were defined as otology fellows at the beginning of their subspecialty training (Jr). Sr surgeons had performed at least 20 micropick fenestrations, whereas Jr surgeons had no experience with micropick fenestration of the stapes footplate.

For evaluating performance, we constructed a surgical model of stapedotomy based on a human temporal bone with instrumentation for quantitative measurement ${ }^{16}$ (Fig 2). In our surgical model of stapedotomy, a prepared human temporal bone is mounted on a ball-swivel joint (Newport Corporation, Irvine, CA), which allows the surgeon to position the temporal bone in a position amenable to transcanal surgery. The temporal bone has been modified to permit positioning medial to the oval window of an ELFS-T3M-L5 load cell (Entran, Fairfield, NJ) to measure forces. The load cell is mounted on 3-Axis Dovetail x-y-z stage (Newport Corporation).

On the load cell, we place an artificial stapes footplate made of dense foam (Pacific Research Laboratories, Inc, Vashon, WA). The force required to fenestrate the stapes footplate varies with thickness. Because of the high variability in thickness of natural bony footplates, we use synthetic artificial stapes footplates of more uniform thickness with mechanical properties similar to natural footplates.

For fenestration of the stapes footplate using a micropick, performance measures are (1) targeting of the fenestration to a desired point and (2) force-both maximum and cumulative-applied to the stapes footplate. Performance variables are measured for $\mathrm{Jr}$ and $\mathrm{Sr}$ operators. We measure performance as follows.

\section{Targeting the Fenestration}

A fenestration target is designated by a submillimeter dot on the artificial footplate. This is captured on a prefenestration image taken using 


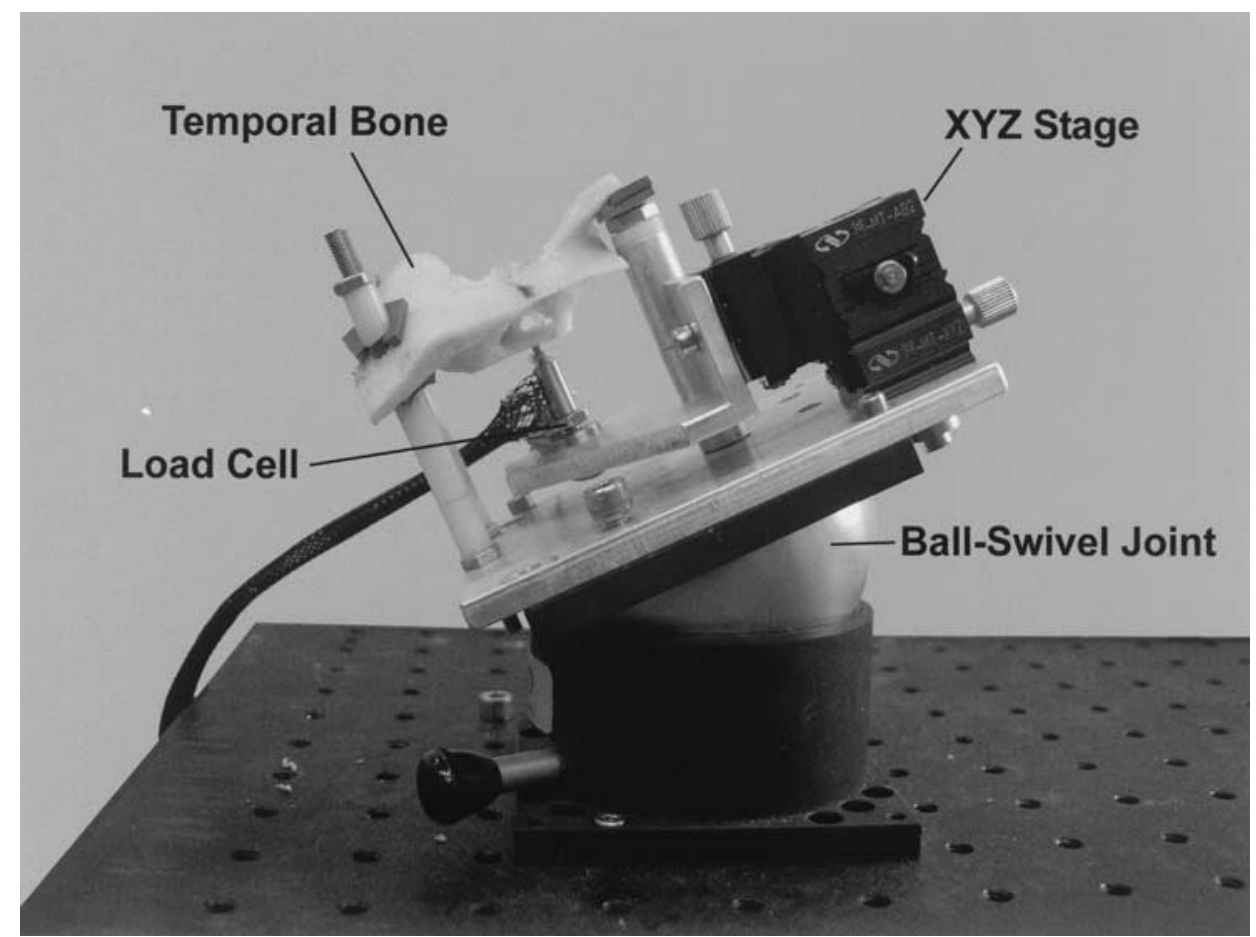

Fig 2. Stapedotomy surgical station.

a digital camera (Camedia E-10 Digital Camera; Olympus, Melville, NY). After fenestration, the footplate is again photographed. Displacement between the resulting images is calculated using a MATLAB (Version 6.0; The MathWorks, Inc, Natick, MA) image-processing program (Fig 3).

\section{Force Applied to the Stapes Footplate}

Forces applied to the stapes footplate are measured using the load cell on which the stapes footplate is mounted. Both the maximum force and cumulative force (area under the curve) applied during fenestration are recorded (Fig 4).

Comparing free-hand $(\mathrm{FH})$ fenestration performance with $\mathrm{SH}$ robot-assisted performance in force-feedback mode primarily measures the effect of dampening tremor on fenestration. Comparing FH performance with robot-assisted performance in force-scaling mode measures the combined effects of force scaling and tremordampening. The $\mathrm{SH}$ robot operates only as a viscous system and therefore the effect of force scaling cannot be compared directly with $\mathrm{FH}$ fenestration independent of tremor dampening.
To become familiar with both the surgical model of stapedotomy and the robot, subjects underwent a series of pretrial training drills. Operators then performed 5 fenestrations under 3 different experimental conditions: (1) free-hand ( $\mathrm{FH})$, that is, no robotic assistance; (2) robotic assistance with 1:1 force-feedback (force-feedback mode); and (3) robotic assistance with 2:1 force scaling (force-scaling mode).

Multiple linear regressions were performed to determine the independent effects of surgeon experience and $\mathrm{SH}$ robotic assistance on performance. Separate regressions compared each robot control mode - force-feedback and force-scaling - with FH performance. Each regression had 2 independent variables - surgical experience ( $\mathrm{Jr} / \mathrm{Sr}$ ) and presence/ absence of robotic assistance. Regressions were performed for each of 4 dependent variables - displacement, maximum force, cumulative force, and duration of fenestration.

Independent variables were dichotomous. For surgeon experience $\left(\mathrm{x}_{1}\right)$, the dichotomous states were defined as $\operatorname{Jr}\left(\mathrm{x}_{1}=0\right)$ and $\operatorname{Sr}\left(\mathrm{x}_{1}=1\right)$ surgeon. For robot assistance $\left(\mathrm{x}_{2}\right)$, the dichoto- 
Before

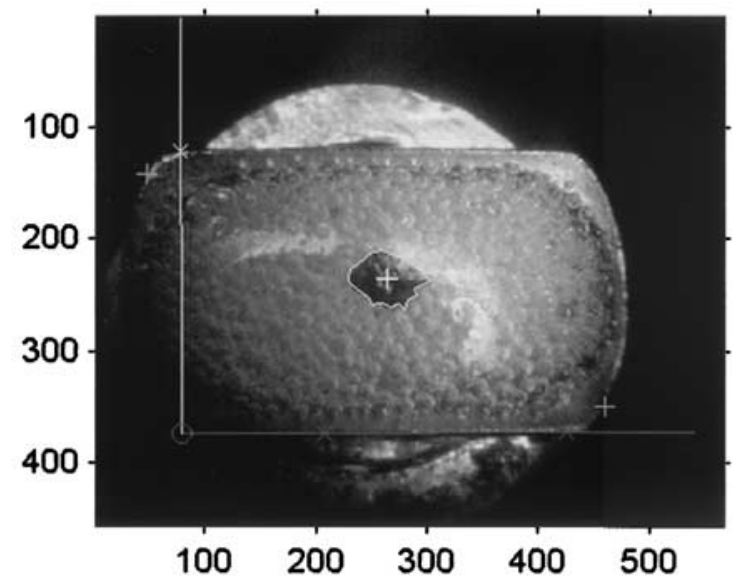

After

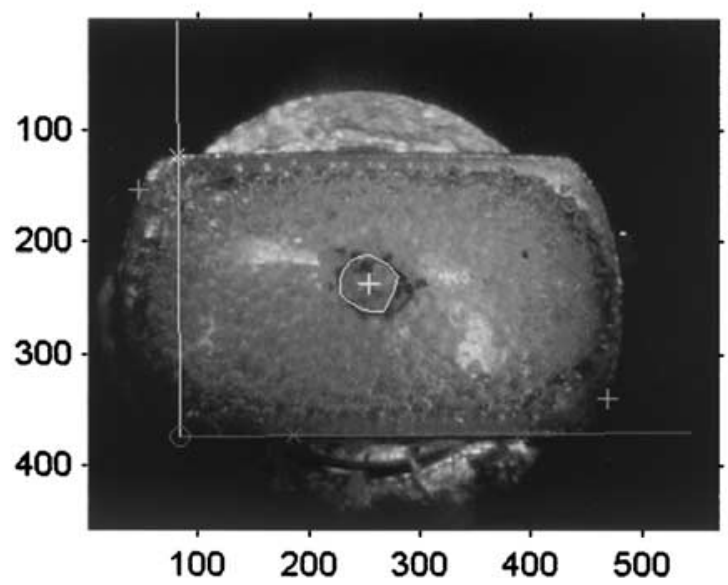

Fig 3. Displacement. To measure fenestration targeting, before and after pictures are used to calculate the displacement between the desired and actual centers of fenestration (crossed lines). Displacement, 0.109 $\mathrm{mm}$.

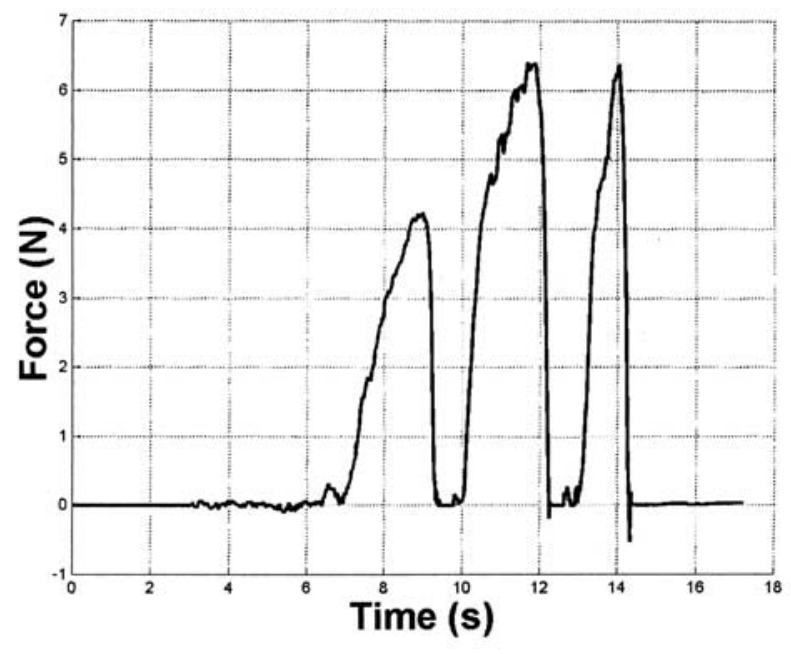

Fig 4. Load cell force versus time. A force sensing load cell positioned beneath the stapes footplate measures forces during fenestration.

mous states were defined as $\mathrm{FH}\left(\mathrm{x}_{2}=0\right)$ and $\mathrm{SH}$ robot-assisted fenestration $\left(\mathrm{x}_{2}=1\right)$.

The coefficient for each independent variable represents the average effect on the dependent variable of changing from one state to the other. Because 2 dichotomous variables $\left(\mathrm{x}_{1}\right.$ and $\left.\mathrm{x}_{2}\right)$ were used in the same multiple linear regression, their coefficients represent independent effects on the dependent variable. Thus the effect of surgeon experience $\left(\mathrm{x}_{1}\right)$ on a given outcome is independent the nature of the fenestration $\left(\mathrm{x}_{2}\right)$, such as $\mathrm{FH}$ or robot assisted.

\section{RESULTS Individual Effects on Group Differences}

To establish if individual differences between surgeons were an important determinant of overall performance, we conducted an ANOVA for each performance variable: displacement, maximum force, and cumulative force. The goal was to establish whether individual surgeons might bias group comparisons (Jr versus $\mathrm{Sr}$ surgeons) by disproportionately affecting group performance. For maximum force applied to the stapes footplate and displacement between the actual and desired centers of fenestration, individual differences between surgeons did not seem to be important predictors of performance (ANOVA $P=0.70$ and 0.61, respectively). However, individual differences between surgeons did significantly affect cumulative force applied to the stapes footplate (ANOVA $P=$ 0.012). Of factors affecting cumulative force, duration of fenestration showed the strongest correlation (Pearson correlation coefficient $=0.83$, such as strongly correlated) and accounted for most of the individual differences in cumulative force. Therefore, differences between $\mathrm{Jr}$ and $\mathrm{Sr}$ surgeons observed for cumulative force may be a result of individual instead of group differences. 
Table 1. Multiple linear regressions for SH robot at 1:1 force-feedback

\begin{tabular}{|c|c|c|c|c|c|c|c|c|}
\hline \multirow[b]{2}{*}{$\begin{array}{c}\text { Binary } \\
\text { independent variable }\end{array}$} & \multicolumn{2}{|c|}{$\begin{array}{l}\text { Displacement } \\
(\mathrm{mm})\end{array}$} & \multicolumn{2}{|c|}{$\begin{array}{l}\text { Maximum force } \\
\text { (N) }\end{array}$} & \multicolumn{2}{|c|}{$\begin{array}{l}\text { Cumulative force } \\
\text { (Ns) }\end{array}$} & \multicolumn{2}{|c|}{$\begin{array}{c}\text { Duration of } \\
\text { fenestration (s) }\end{array}$} \\
\hline & $\begin{array}{l}\text { Coefficient } \\
(\mathrm{mm})\end{array}$ & $P$-value & $\begin{array}{l}\text { Coefficient } \\
\text { (N) }\end{array}$ & $P$-value & $\begin{array}{l}\text { Coefficient } \\
\text { (Ns) }\end{array}$ & $P$-value & $\begin{array}{l}\text { Coefficient } \\
\text { (s) }\end{array}$ & $P$-value \\
\hline \multicolumn{9}{|l|}{$\mathrm{X}_{1}:$ Experience } \\
\hline $\begin{array}{l}\text { Junior surgeon }\left(X_{1}=0\right) \\
\text { Senior surgeon }\left(X_{1}=1\right)\end{array}$ & -0.04 & 0.27 & 0.05 & 0.93 & -8.57 & 0.59 & 3.66 & 0.54 \\
\hline \multicolumn{9}{|l|}{$\mathrm{X}_{2}:$ Robot assistance } \\
\hline $\begin{array}{l}\mathrm{FH}\left(\mathrm{X}_{2}=0\right) \\
\text { SH 1:1 }\left(\mathrm{X}_{2}=1\right)\end{array}$ & -0.02 & 0.48 & -0.36 & 0.50 & -31.11 & 0.05 & -12.67 & 0.04 \\
\hline
\end{tabular}

\section{SH Robot Assistance in Force- Feedback Mode: Tremor Reduction}

Table 1 presents the results of multiple linear regressions comparing $\mathrm{FH}$ performance to $\mathrm{SH}$ robot assistance in force-feedback mode. In this mode, the primary effect of SH robotic assistance is to reduce tremor. Table 1 shows that the $\mathrm{SH}$ robot in force-feedback mode significantly reduced the cumulative force applied to the stapes footplate by an average of 31 Ns $(P=0.05)$. This is a reduction of approximately $58 \%$. Cumulative force was not significantly affected by surgeon experience. Neither surgeon experience nor $\mathrm{SH}$ robotic assistance in force-feedback mode affected maximum force or fenestration targeting (displacement).

Both cumulative force and duration of fenestration are significantly reduced by $\mathrm{SH}$ robot assistance in force-feedback mode (Table 1). Therefore, SH robotic assistance in force-feedback mode reduces cumulative force at the stapes footplate likely by decreasing the duration of fenestration.

\section{SH Robot Assistance in Force-Scaling Mode: Tremor Reduction and Amplified Tactile Feedback}

Table 2 presents the results of multiple linear regressions comparing $\mathrm{FH}$ performance to $\mathrm{SH}$ robotic assistance in force-scaling mode. In this mode, $\mathrm{SH}$ robotic assistance provides both tremor reduction and amplified tactile feedback. Table 2 shows that $\mathrm{SH}$ robot assistance in force-scaling mode significantly reduced the maximum force applied to the stapes footplate by an average of
$1.08 \mathrm{~N}(P=0.04)$. This is a reduction of approximately $17 \%$. Neither surgeon experience nor robotic assistance in force-scaling mode significantly affected fenestration targeting (displacement).

In force-scaling mode, $\mathrm{SH}$ robot assistance also reduced cumulative force applied to the stapes footplate by an average of $22 \mathrm{Ns}$, a reduction of $30 \%$. However, unlike in force-feedback mode, the effect did not reach statistical significance $(P=0.17)$. The reason for this difference resides in the fact that cumulative force is primarily a function of duration of fenestration. Because robotic assistance in force-scaling mode did not reduce duration of fenestration to a statistically significant degree $(P=0.16)$, neither did it significantly reduce cumulative force.

\section{Surgeon Experience and SH Robot Assistance}

Using SH robot assistance, there were no significant differences in any performance measure between $\mathrm{Jr}$ and $\mathrm{Sr}$ surgeons (Table 3). Table 3 compares mean performance measures for $\mathrm{Jr}$ and $\mathrm{Sr}$ surgeons using robot assistance in force-feedback mode. The same results occurred for robot assistance in force-scaling mode (data not shown).

In both control modes (force-feedback and force-scaling), $\mathrm{SH}$ robot assistance significantly improved fenestration targeting, that is, reduced displacement, for Jr surgeons (Tables 4 and 5). In contrast, $\mathrm{SH}$ robot assistance significantly reduced the ability of Sr surgeons to target the fenestration, increasing displacement by approximately $80 \%$ (Tables 6 and 7). No statistically significant effects on either maximum or cumulative force were seen. 
Table 2. Multiple linear regressions for $\mathrm{SH}$ robot at 2:1 force scaling

\begin{tabular}{|c|c|c|c|c|c|c|c|c|}
\hline \multirow[b]{2}{*}{$\begin{array}{c}\text { Binary } \\
\text { independent variable }\end{array}$} & \multicolumn{2}{|c|}{$\begin{array}{l}\text { Displacement } \\
(\mathrm{mm})\end{array}$} & \multicolumn{2}{|c|}{$\begin{array}{l}\text { Maximum force } \\
(\mathrm{N})\end{array}$} & \multicolumn{2}{|c|}{ Cumulative force (Ns) } & \multicolumn{2}{|c|}{$\begin{array}{c}\text { Duration of } \\
\text { fenestration (s) }\end{array}$} \\
\hline & $\begin{array}{l}\text { Coefficient } \\
\text { (mm) }\end{array}$ & $P$-value & $\begin{array}{l}\text { Coefficient } \\
\text { (N) }\end{array}$ & $P$-value & $\begin{array}{l}\text { Coefficient } \\
\text { (Ns) }\end{array}$ & $P$-value & $\begin{array}{l}\text { Coefficient } \\
\text { (s) }\end{array}$ & $P$-value \\
\hline $\begin{array}{l}X_{1} \text { : Experience } \\
\quad \text { Junior surgeon }\left(X_{1}=0\right) \\
\text { Senior surgeon }\left(X_{1}=1\right)\end{array}$ & -0.04 & 0.25 & 0.01 & 0.98 & -6.92 & 0.66 & 4.74 & 0.41 \\
\hline $\begin{array}{l}\mathrm{X}_{2}: \text { Robot assistance } \\
\text { FH }\left(\mathrm{X}_{2}=0\right) \\
\text { SH } 2: 1\left(\mathrm{X}_{2}=1\right)\end{array}$ & -0.02 & 0.60 & -1.08 & 0.04 & -22.09 & 0.17 & -8.11 & 0.16 \\
\hline
\end{tabular}

Table 3. Comparison of performance using SH in force-feedback mode

\begin{tabular}{lccc}
\hline & $\begin{array}{c}\text { Junior } \\
\text { surgeon }\end{array}$ & $\begin{array}{c}\text { Senior } \\
\text { surgeon }\end{array}$ & P-value \\
\hline Displacement (mm) & 0.118 & 0.177 & 0.12 \\
Maximum force $(\mathrm{N})$ & 6.19 & 5.99 & 0.77 \\
Cumulative force $(\mathrm{Ns})$ & 48.43 & 28.74 & 0.21 \\
\hline
\end{tabular}

Table 4. Junior surgeon comparison for $\mathrm{SH}$ in force-feedback mode

\begin{tabular}{lccc}
\hline & FH & SH 1:1 & P-value \\
\hline Displacement (mm) & 0.236 & 0.118 & 0.01 \\
Maximum force (N) & 6.30 & 6.19 & 0.86 \\
Cumulative force (Ns) & 68.76 & 48.43 & 0.37 \\
Duration of fenestration (s) & 25.51 & 18.88 & 0.16 \\
\hline
\end{tabular}

Table 5. Junior surgeon comparison for $\mathrm{SH}$ in force-scaling mode

\begin{tabular}{lccc}
\hline & FH & SH 2:1 & P-value \\
\hline Displacement (mm) & 0.236 & 0.135 & 0.04 \\
Maximum force (N) & 6.30 & 5.46 & 0.25 \\
Cumulative force (Ns) & 68.76 & 54.87 & 0.55 \\
Duration of fenestration (s) & 25.51 & 21.85 & 0.29 \\
\hline
\end{tabular}

\section{DISCUSSION}

Robotic assist devices have the potential to improve surgical dexterity through enhanced stability, precision and tactile feedback. The goal is to improve current surgical treatments and to enable new operative procedures. Like other technologic innovations, such as the operating microscope, robots may improve otologic surgery by overcoming basic human limitations. However, developing
Table 6. Senior surgeon comparison for $\mathrm{SH}$ in force-feedback mode

\begin{tabular}{lccc}
\hline & FH & SH 1:1 & P-value \\
\hline Displacement (mm) & 0.100 & 0.177 & 0.05 \\
Maximum force (N) & 6.60 & 5.99 & 0.49 \\
Cumulative force (Ns) & 71.53 & 28.74 & 0.10 \\
Duration of fenestration (s) & 35.51 & 16.30 & 0.04 \\
\hline
\end{tabular}

Table 7. Senior surgeon comparison for $\mathrm{SH}$ in force-scaling mode

\begin{tabular}{lccc}
\hline & FH & SH 2:1 & P-value \\
\hline Displacement (mm) & 0.100 & 0.186 & 0.01 \\
Maximum force (N) & 6.60 & 5.22 & 0.11 \\
Cumulative force (Ns) & 71.53 & 39.46 & 0.21 \\
Duration of fenestration (s) & 35.51 & 21.97 & 0.11 \\
\hline
\end{tabular}

robotic assist devices for otologic surgery and identifying the optimal procedures for their application will require continued experimentation. That process involves first defining difficult surgical tasks that might benefit from robotic assistance and then assessing robotic applications by comparing performance to conventional $\mathrm{FH}$ techniques.

\section{SH Robotic Assistance in Force- Feedback Mode: Tremor Reduction}

The primary effect of $\mathrm{SH}$ robot assistance in force-feedback mode is to reduce tremor. For micropick fenestration, tremor reduction significantly affected 2 performance variables: (1) cumulative force applied to the stapes footplate and (2) fenestration targeting.

$\mathrm{SH}$ robotic assistance in force-feedback mode significantly reduced the cumulative force applied 
to the stapes footplate. The effect seemed to be mediated by reductions in the duration of fenestration. The mechanism by which tremor reduction decreases duration of fenestration is uncertain; perhaps the steadying nature of the system makes operators more confident in the precise movements of fenestration.

Interestingly, reduction in duration of fenestration with robotic assistance is more pronounced without amplified tactile feedback. Adding force scaling to tremor reduction blunts the time-shortening effect of tremor reduction alone. As a result, unlike in force-feedback mode, the effect of SH robot assistance in force-scaling mode on cumulative force was not statistically significant.

We surmise that the reason for the negative impact of enhanced tactile feedback on duration of fenestration is that the surgeon must apply twice the handle force to produce the same tip force obtained without force scaling. The user may sense these higher handle forces as "dangerous," and the resulting hesitancy may increase the duration of fenestration compared with tremor reduction without force amplification.

Tremor reduction also significantly affected fenestration targeting. Because fenestration targeting occurs as the surgeon positions the instrument before making contact with the footplate, enhanced tactile feedback should not affect displacement. Indeed, the same dichotomous effect on fenestration targeting was seen for both robotcontrol modes. In both force-feedback and forcescaling modes, SH robot assistance significantly improved fenestration targeting for $\mathrm{Jr}$ surgeons (Tables 4 and 5) and significantly reduced targeting ability for Sr surgeons (Tables 6 and 7).

The reason for a dichotomous effect of robot assistance on the targeting ability of surgeons of different experience levels is uncertain. We surmise that, with repetition and experience, surgeons develop a motor program for micropick fenestration of the stapes footplate. For FH fenestration, this routine allows $\mathrm{Sr}$ surgeons to outperform their less-experienced counterparts (FH displacement, $0.100 \mathrm{~mm}$ versus $0.236 \mathrm{~mm} ; P=0.01$ ). This same routine may also interfere with the ability of $\mathrm{Sr}$ surgeons to learn the modifications in technique that come with robot assistance.
These observations are reminiscent of findings from basic research on motor memory. When learning a new motor task, individuals develop an internal model of task kinematics. This model functions as a template for motor memory and informs future task performance. However, in certain situations, internal kinematic models can interfere with subsequent task learning. Individuals may inappropriately apply existing internal kinematic models to new tasks, and performance may be poor as a result. Interference has been shown to be most manifest in the 4- to 5-hour interval between motor learning and completed memory consolidation. ${ }^{17}$

There are no data in the literature demonstrating postconsolidation interference. Nevertheless, there is good evidence that prior experience with related tasks affects the performance of new tasks. For example, Braille readers develop internal kinematic models for touch perception that seem to improve performance in related tasks. Investigations of active touch perception used in Braille reading indicate that experienced readers significantly outperform inexperienced readers, though with practice the performance of both groups equalizes..$^{18}$

\section{SH Robotic Assistance in Force-Scaling Mode: Tremor Reduction and Enhanced Tactile Feedback}

We also compared $\mathrm{SH}$ robotic assistance in force-scaling mode with $\mathrm{FH}$ fenestration. In this case, $\mathrm{SH}$ robotic assistance provides the surgeon with both tremor reduction and enhanced tactile feedback.

Robotic assistance in force-scaling mode significantly reduced the maximum force applied to the stapes footplate during micropick fenestration compared with FH fenestration. This effect was not seen in force-feedback mode, ie, tremor reduction without enhanced tactile feedback.

At first, the effect of force scaling on maximum force may seem counter-intuitive. One might think that for a given object, such as a micropick, the force to puncture a material is an inherent property of the material, independent of the means of puncture, such as FH or robot assisted.

In our experimental setup, there are only 2 surfaces with which the instrument tip makes con- 
tact: the stapes footplate and the metal surface of the load cell located $0.6 \mathrm{~mm}$ below the bottom surface of the footplate. Therefore, maximum force is a function of contact between the instrument tip and 1 of those 2 surfaces.

Maximum force is not likely a function of micropick contact with the stapes footplate. Reductions in maximum force with $\mathrm{SH}$ robotic assistance were observed only in the presence of both enhanced tactile feedback and tremor reduction. $\mathrm{SH}$ robotic assistance with tremor reduction alone did not significantly reduce maximum force. Because each surgeon fixed the robot's orientation before starting trials and left the robot in that orientation during all robotic trials, the addition of enhanced tactile feedback could only have affected the rate of fenestration through the footplate. However, in subsequent controlled trials, maximum force did not vary by tip velocity during fenestration (data not shown).

The likely scenario then is that the lower maximum force is a function of micropick contact with the metal surface of the load cell. In both FH and $\mathrm{SH}$ robot-assisted fenestration, operators seem to have difficulty sensing when the micropick has completely fenestrated the footplate. During fenestration, users seem to advance the pick until resistance from the metal surface of the load cell increases tip forces to palpable levels. By amplifying the tip force, robot assistance appears to help the operator sense complete fenestration at lower tip contact forces, about $17 \%$ lower.

In stapedotomy, there are no data describing how surgeons know when to halt micropick advancement during fenestration. Our data support the intuitive notion that surgeons stop when they sense a change in resistance. In our experimental model, the change in resistance seems to involve contact with the metal surface of the load cell. As described by senior surgeons, there is a "pop" that occurs during actual stapedotomy when the footplate has been fully fenestrated. The nature of the resistance change causing this "pop" has never been investigated.

The surgical significance of a $17 \%$ reduction in the maximum force applied during micropick fenestration is unknown. In our model, maximum force seems to be a result of micropick contact with structures underlying the footplate. If the same were to be true during actual surgery, then a $17 \%$ reduction in force applied to structures, such as the utricle, saccule, and cochlea, could be quite significant, particularly in terms of reducing complications associated with stapedotomy. Nevertheless, the relationship between forces applied during micropick fenestration and inner ear damage is uncertain.

\section{Understanding Shortcomings in SH Robotic Assistance}

For fenestration targeting as measured by displacement, $\mathrm{SH}$ robotic assistance at its best, that is in the hands of $\mathrm{Jr}$ surgeons, does not outperform $\mathrm{Sr}$ surgeons doing FH micropick fenestration. The obvious conclusion is that for this application the theoretical advantages of tremor reduction do not enhance surgical dexterity beyond that of a $\mathrm{Sr}$ surgeon.

However, before concluding that tremor reduction via proportional velocity control does not improve surgical dexterity for fenestration, other possibilities must be excluded. In its current mechanical coniguration, the $\mathrm{SH}$ robot has clear ergonomic limitations. The end-effector arm is bulky. As a result, it obstructs the surgeon's lineof-sight and requires that the surgeon modify the angle of approach to the footplate. Further optimizing the mechanical coniguration of the robot would permit firmer conclusions to be reached regarding $\mathrm{SH}$ robot limitations.

\section{Interpreting Results: Limitations in Generalizability}

These trials were carried out in a surgical model of stapedotomy. The model approximates the conditions of stapedotomy, but it is not an exact replication of the actual procedure. Furthermore, only $3 \mathrm{Jr}$ and $3 \mathrm{Sr}$ surgeons participated in these trials. Therefore, conclusions derived from trials with this model should be considered preliminary.

In our surgical model of stapedotomy, the maximum force required to fenestrate a synthetic stapes footplate was approximately $6 \mathrm{~N}$. Natural fenestration forces are between 5 and $8 \mathrm{~N}$. However, these data were derived during fenestration with a microdrill. ${ }^{19}$ Force data for micropick fenestration has yet to be reported. 


\section{Robotic Assistance: Targeting Applications}

One of the important issues in developing robotic assist devices is to identify the ways in which different robot capabilities affect surgical performance. For example, we found that the $\mathrm{SH}$ robot significantly reduced the force applied to the stapes footplate during fenestration but had ambiguous effects on fenestration targeting that were dependent on surgeon experience.

A better understanding of robot capabilities can inform decisions regarding future applications of robotics to surgery. For micropick fenestration, we demonstrated a significant reduction in maximum fenestration force using $\mathrm{SH}$ robotic assistance with amplified force feedback. Nevertheless, enhanced tactile feedback might be even more useful for microdrilling of the stapes footplate. Unlike micropick fenestration, drilling works primarily via shearing forces. Thus, robotic assistance with enhanced tactile feedback might be able to limit the normal force applied to the footplate during drilling to almost zero, offering much better protection for the inner ear.

Furthermore, a better understanding of the strengths of robotic assistance might permit the development of new surgical procedures beyond conventional human capabilities but well suited to robotic assistance. For instance, by permitting precise, stable positioning of surgical instruments, robot assist devices might permit needle placement in the inner ear for the targeted delivery of therapy to treat conditions such as hearing loss and vertigo.

We gratefully acknowledge the following individuals for their assistance: David Ryugo, Sam Lang, Terry Shelley, Jay Burns, Jason Wachs, Aaron Barnes, Robert Cammarata, Ingrid Shao, Han Seo Cho, and Matthew Hansen.

\section{REFERENCES}

1. Schuknecht HF. Stapedectomy. 1st ed. Boston: Little, Brown and Company; 1971.

2. Riviere CN, Rader RS, Khosla PK. Characteristics of hand motion of eye surgeons. Presented at the Proceedings of the 19th Annual International Conference of the IEEE in Medicine and Biology Society; October 30November 2, 1997; Chicago, IL.

3. Gupta PK, Jensen PS, deJuan E. Surgical forces and tactile perception during retinal microsurgery. Paper presented at Medical Image Computing and Computer-As- sisted Interventions (MICCAI); September 19-22, 1999; Cambridge, England.

4. Charles S. Dexterity enhancement for surgery. In: Taylor RH, Lavallee S, Burdea GC, et al, eds. Computer Integrated Surgery: Technology and Clinical Applications. Cambridge, Massachussetts: MIT Press; 1996. p. 467-71.

5. Kaburlasos VG, Petridis V, Brett PN, et al. Estimation of the stapes-bone thickness in the stapedotomy surgical procedure using a machine-learning technique. IEEE Trans Inf Technol Biomed 1999;3:268-77.

6. Brett PN, Baker DA, Reyes L, et al. An automatic technique for micro-drilling a stapedotomy in the flexible stapes footplate. Proc Inst Mech Eng [H] 1995;209:25562.

7. Kazerooni H, Guo J. Human extenders. Trans ASME J Dyn Systems Measurement Control 1989;115:281-90.

8. Ho SC, Hibberd RD, Davies BL. Robot assisted knee surgery. IEEE Eng Med Biol 1995;14:292-300.

9. Taylor RH, Jensen PS, Whitcomb LL, et al. A steadyhand robotic system for microsurgical augmentation. Int J Robot Res 1999;18:1201-10.

10. Kumar R, Goradia T, Barnes A, et al. Performance of Robotic Augmentation in Microsurgery-Scale Motions. Paper presented at Lecture Notes in Computer Science 1679: Medical Image Computing and Computer-Assisted Intervention-MICCAI 99: the Second International Conference; September 19-22, 1999; Cambridge, England. p. 1108-15.

11. de Bruijn AJ, Tange RA, Dreschler WA. Efficacy of evaluation of audiometric results after stapes surgery in otosclerosis. I. The effects of using different audiologic parameters and criteria on success rates. Otolaryngol Head Neck Surg 2001;124:76-83.

12. Sedwick JD, Louden CL, Shelton C. Stapedectomy vs stapedotomy. Do you really need a laser? Arch Otolaryngol Head Neck Surg 1997;123:177-80.

13. Hughes GB. The learning curve in stapes surgery. Laryngoscope 1991;101(12 Pt 1):1280-4.

14. Levenson MJ. Methods of teaching stapedectomy. Laryngoscope 1999;109:1731-9.

15. Mathews SB, Hetzler DG, Hilsinger RL Jr. Incus and stapes footplate simulator. Laryngoscope 1997;107(12 Pt 1):1614-6.

16. Berkelman P, Rothbaum DL, Roy J, et al. Performance evaluation of a cooperative manipulation microsurgical assistant robot applied to stapedotomy. Paper presented at Lecture Notes in Computer Science: Medical Image Computing and Computer-Assisted Intervention MICCAI, The Fourth International Conference; October, 2001; Utrecht, the Netherlands. p. 1426-28.

17. Shadmehr R, Brashers-Krug T. Functional stages in the formation of human long-term motor memory. J Neurosci 1997;17:409-19.

18. Grant AC, Thiagarajah MC, Sathian K. Tactile perception in blind Braille readers: a psychophysical study of acuity and hyperacuity using gratings and dot patterns. Percept Psychophys 2000;62:301-12.

19. Khodabandehloo K, Brett PN, Buckingham RO. Specialpurpose actuators and architecture for surgery robots. In: Taylor RH, Lavallee S, eds. Computer Integrated Surgery: Technology and Clinical Applications. London: MIT Press; 1996. p. 263-76. 